\title{
Neutron Scattering Measurements of the Phonon Density of States of $\mathrm{FeSe}_{1-x}$ Superconductors
}

\author{
D. Phelan ${ }^{1}$, J. N. Millican ${ }^{1}$, E. L. Thomas ${ }^{2}$, J. B. Leão ${ }^{1}$, Y. Qiu ${ }^{1,3}$, R. Paul ${ }^{1}$ \\ ${ }^{1}$ NIST Center for Neutron Research, \\ National Institute of Standards and Technology, \\ Gaithersburg, Maryland 20899, USA \\ ${ }^{2}$ Ceramics Division, National Institute of Standards and Technology, \\ Gaithersburg, Maryland 20899, USA \\ ${ }^{3}$ Department of Materials Science and Engineering, \\ University of Maryland, College Park, Maryland 20742, USA
}

(Dated: November 4, 2018)

\begin{abstract}
Inelastic neutron-scattering experiments have been carried out on polycrystalline samples of the $\mathrm{FeSe}_{1-x}$ superconductors. We report the phonon density of states for $\mathrm{FeSe}_{1-x}$ with $\mathrm{Tc} \approx 8 \mathrm{~K}$. The phonon cutoff frequency is observed around $40 \mathrm{meV}$. No significant change is observed across the superconducting transition. The measurements support the published first-principles calculations [A. Subedi et al., Phys. Rev. B 78, 134514 (2008)].
\end{abstract}




\section{INTRODUCTION}

Recently, the family of Fe superconductors, which had consisted of only compounds containing both Fe and As, has been expanded to include $\mathrm{Fe}(\mathrm{Se} / \mathrm{Te})_{1-x}$ [1]. $\mathrm{A} \mathrm{T}_{c}$ of approximately $8 \mathrm{~K}$ has been reported for $\mathrm{FeSe}_{1-x}$ at ambient pressure [1], while a $\mathrm{T}_{c}$ of 15 $\mathrm{K}$ has been reported in $\mathrm{Fe}\left(\mathrm{Se}_{0.5} \mathrm{Te}_{0.5}\right)_{1-x}\left[2,[3]\right.$. Interestingly, $\mathrm{T}_{c}$ is very sensitive to pressure and increases to $27 \mathrm{~K}$ by the application of $1.48 \mathrm{GPa}$ in FeSe [4]. The planar features of the crystal structure of $\mathrm{Fe}(\mathrm{Se} / \mathrm{Te})_{1-x}$, which is $\mathrm{PbO}$ type, are similar to those of the FeAs compounds. Moreover, first-principles calculations of the band structure [5] of FeSe show analogous features in the Fermi surface to LaOFeAs. This suggests that the class of $\mathrm{Fe}(\mathrm{Se} / \mathrm{Te})_{1-x}$ materials may play an important role in elucidating the nature of the superconductivity of FeAs-based superconductors.

While the mechanism of superconductivity in $\mathrm{Fe}(\mathrm{Se} / \mathrm{Te})_{1-x}$ compounds is yet unknown, the field is developing quickly. Initially it was believed that a nonstoichiometric ratio (i.e., not 1:1) of Fe:Se was critical for the superconductivity [1] and that this nonstoichiometry resulted in anion vacancies, which could have the effect of stabilizing magnetic clusters [6]. Very recently a report has appeared that suggests that for the Te-free compounds superconductivity only appears in a narrow compositional range near perfectly stoichiometric FeSe [7]. No coherence peak, which would be typical for a phonon-mediated $s$-wave superconductor, is observed in NMR measurements [8]. A possible alternative to a phonon-mediated mechanism is an unconventional magnetic mechanism related to robust incommensurate spin correlations [9].

In this paper, we report the results of inelastic neutronscattering measurements performed on powder samples of $\mathrm{FeSe}_{1-x}$, which were made to determine the phonon density of states (PDOS) at several temperatures. The PDOS is an important quantity for superconducting compounds because, according to standard electron-phonon coupling theory, $\mathrm{T}_{c}$ is related to the spectral weight of phonon vibrations. Moreover, it is a quantity that can be directly compared to theoretical calculations, and we show that there is a fair agreement between the first-principles calculations of Subedi et al. [5] and the current measurements. 


\section{EXPERIMENT}

Polycrystalline $\mathrm{FeSe}_{1-x}$ samples were synthesized by solid-state reaction from elemental Fe and Se powders. All handling was performed in an inert He glove box in which a very low humidity level (less than $35 \mathrm{ppm}$ ) was maintained so that, once the synthesis began, the samples measured in the inelastic neutron-scattering experiments were never exposed to the atmosphere. Initially, the desired quantities of Fe and Se were ground together and then put in a domed quartz tube connected to a closed valve. The tube was then placed in a tube furnace and heated to $110{ }^{\circ} \mathrm{C}$ with the valve opened to evacuate for an hour in order to remove any gas or moisture. The valve was then closed to minimize deposition of Se on the cold end of the tube and in the vacuum line, and the sample was heated to 670 ${ }^{\circ} \mathrm{C}$ for $13 \mathrm{~h}$ in vacuum and then cooled back down to room temperature. The sample was reground, placed back in the tube, and a similar process was carried out, except that this time, the sample was heated to $670{ }^{\circ} \mathrm{C}$ for $24 \mathrm{~h}$ and then the temperature was lowered to $400{ }^{\circ} \mathrm{C}$, which was maintained for $36 \mathrm{~h}$. The last step was critical for avoiding the NiAs phase of FeSe.

The motivation for handling the powders for the inelastic neutron-scattering measurements in an inert environment was to avoid hydrogen contamination in the samples due to contact with air or moisture from the air. Hydrogen contamination is undesirable for inelastic neutron-scattering measurements due to the large incoherent-scattering cross section of hydrogen. To determine the hydrogen content in the samples, sample B (see below) was analyzed by prompt gamma activation analysis (PGAA), which is a nondestructive technique for determining accurate quantities of hydrogen and other elements, on beamline NG-7 at the NIST Center for Neutron Research (NCNR) [10, 11]. Samples that had been handled in air exhibited a $\mathrm{Fe} / \mathrm{H}$ molar ratio of approximately ten; however, the sample grown in the glove box had a significantly improved ratio of approximately 28 . Relative $1 \sigma$ uncertainties for the latter PGAA measurements were $<10 \%$, based on counting statistics.

Using the procedure outlined above, two samples were grown: one (sample A) with an initial Fe:Se ratio of 1.0:0.72 and the other (sample B) with a ratio of 1.0:0.82. Approximately $14 \mathrm{~g}$ of sample A and $12 \mathrm{~g}$ of sample B were sealed in aluminum cans with indium gaskets while in the glove box for the inelastic neutron-scattering experiments. A remaining small quantity of both samples was also removed from the glove box for x-ray diffraction mea- 
surements and an electrical resistivity measurement. X-ray diffraction measurements were made using a commercial diffractometer with $\mathrm{Cu} \mathrm{K \alpha}$ radiation $(\lambda=1.5405 \AA)$. As shown in Fig. 1(a), both samples had very clean x-ray patterns except for a few very small potential impurity peaks which are labeled. The resistivity of sample A, shown in Fig. 1(b), was measured on a pellet that had been pressed and heated at $400{ }^{\circ} \mathrm{C}$ for $12 \mathrm{~h}$. The sample undergoes a superconducting transition at $8 \mathrm{~K}$. Our previous measurements of a sample grown with the same initial starting materials as sample B indicated a superconducting transition at practically the same temperature.

There are three models for the sample stoichiometry of superconducting $\mathrm{Fe}(\mathrm{Se} / \mathrm{Te})_{1-x}$ samples based on Rietveld refinements of powder neutron and x-ray diffraction data. In one model [12], there are vacancies on anion sites. According to Refs. [9] and [13], anion sites are fully occupied and additional Fe atoms occupy interstitial sites in nonstoichiometric mixed Se/Te compositions. Finally, according to Ref. [7], superconducting Te-free samples are actually nearly stoichiometric, and the additional Fe used in the synthesis process actually forms impurities that are difficult to detect by x ray. Given these differing results, we performed powder neutron diffraction measurements and carried out Rietveld refinement on our samples in order to determine their stoichiometry. Powder neutron-diffraction measurements were performed on sample B on the BT-1 powder diffractometer at the NCNR with $\lambda=2.0782 \AA$ obtained from a Ge(311) monochromator, and Rietveld refinement was carried out using GSAS (Ref. [14]) with EXPGUI interface [15]. The refinement of sample B at 298 $\mathrm{K}$ in tetragonal (P4/nmms space group) is shown in Fig. 2(a), where it is clear that there is a very good agreement between the observed and calculated intensities, and the fitted parameters are listed in Table I. Consistent with the report of Ref. [7], we found that our sample was nearly stoichiometric. Again, consistent with Ref. [7], the placement of Fe on interstitial sites worsened the fit. The measurement at $4 \mathrm{~K}$ indicated that a structural phase transition had occurred, and the lower temperature phase was identified as orthorhombic (space group Cmme), consistent with the report of Ref. [12]. The Rietveld refined pattern is shown in Fig. 2(b), and the refined positions are listed in Table I. Our data indicates that the structural phase-transition temperature was between 60 and $80 \mathrm{~K}$, which is again consistent with Ref. [12]. Additionally, Rietveld refinement was carried out on sample A at $300 \mathrm{~K}$ using the diffraction data measured on the disk chopper spectrometer (DCS) at the NCNR with $\lambda=1.8 \AA$ (more details of the DCS measurement are provided below). Similarly, 
the best refinement showed the sample was nearly stoichiometric (the best fit was FeSe.99). As it appears that samples A and B have essentially the same stoichiometry, it is clear that sample A has a larger content of Fe-based impurities, such as $\mathrm{Fe}, \mathrm{Fe}_{3} \mathrm{O}_{4}$, and $\mathrm{Fe}_{3} \mathrm{Si}$ than sample B. As pointed out in Ref. [7], such impurities are not easily detected by x-ray diffraction but are much easier to see by neutron diffraction, which explains why the x-ray patterns in Fig. 1 are clean. The overlap of Fe and Al Bragg reflections in our neutron-scattering experiments makes it impossible to determine the precise phase fractions of $\mathrm{Fe}$ or $\mathrm{Fe}_{3} \mathrm{Si}$ in the sample but, since the refinement of occupancies in FeSe strongly indicates the sample is nearly stoichiometric, it can be inferred that the additional Fe atoms form impurities. As discussed in the following section, a comparison of the inelastic spectra of samples A and B gives an indication that the scattering from such impurities in the PDOS is not substantial.

Inelastic neutron-scattering measurements were made on the BT-4 filter-analyzer spectrometer (FANS) at the NCNR. The operational principle of the measurement is described in Ref. [16]. A pyrolitic graphite (PG) (002) monochromator was used for measurements of energy transfer, $\hbar \omega=\mathrm{E}_{i}-\mathrm{E}_{f}$ (where $\mathrm{E}_{i}$ and $\mathrm{E}_{f}$ are the incident and scattered-neutron energies, respectively), from 5 to $44 \mathrm{meV}$ with collimations of 40'-40' (in pilepost monochromator), giving an energy resolution that varied from $1.1 \mathrm{meV}$ full width at half maximum (FWHM) at $\hbar \omega=5$ to $3.9 \mathrm{meV}$ at $\hbar \omega=44 \mathrm{meV}$. A Cu(220) monochromator was used for measurements with $\hbar \omega$ from 35 to $107 \mathrm{meV}$ with collimations of 40'-40', giving an energy resolution that varied from $1.5 \mathrm{meV} F W H M$ at $\hbar \omega=44$ to $5 \mathrm{meV}$ at $\hbar \omega=107 \mathrm{meV}$. The sample was measured while in a top-loading closed cycle refrigerator with a base temperature of $3.8 \mathrm{~K}$. Cadmium masks covered both the top and bottom flanges of the aluminum can to minimize the background. The inelastic measurements were repeated on an identical empty sample can to determine the background.

Additional inelastic neutron-scattering measurements at lower values of $\hbar \omega$ were performed on DCS at the NCNR [17]. Measurements were made with incident wavelengths of $1.8,2.9,4.8$, and $7.0 \AA$, although all the data shown here were taken with $\lambda=1.8 \AA$. The sample was measured in a He cryostat with a base temperature of $1.5 \mathrm{~K}$ in the same aluminum sample can used for FANS. The DAVE software package was used for elements of the data reduction and analysis [18]. 


\section{RESULTS AND DISCUSSION}

For a measurement on FANS, the scattered neutron energy is chosen by a series of filters that only allow neutrons in a narrow band with $<E_{f}>=1.2 \mathrm{meV}$ to pass through [19]. After subtracting out the background, the measured intensity, $I(\omega)$ is approximately proportional to the neutron-weighted PDOS [16], $G(\omega)$, given by [20]:

$$
G(\omega)=\sum_{i} \sigma_{i} \exp \left(-2 W_{i}\right) G_{i}(\omega) / m_{i}
$$

where the sum occurs over the different atomic species - in this case, Fe and Se. $\sigma_{i}, \mathrm{~m}_{i}$, and $\mathrm{W}_{i}$ are the neutron scattering cross-section, the atomic mass, and the Debye-Waller factor for atom species $i$. The incoherent approximation is made so that the $\sigma_{i}$ are the total (sum of incoherent and coherent) cross-sections, which are 11.62 barns and 8.30 barns for Fe and Se, respectively. Thus, the $\frac{\sigma}{m}$ weighting is $1.98-1$ for Fe to Se. $G_{i}(\omega)$ is the partial weighted PDOS defined by:

$$
G_{i}(\omega)=\frac{1}{3 N} \sum_{j, \vec{k}}\left|\vec{e}_{i}(j, \vec{k})\right|^{2} \delta[\omega-\omega(j, \vec{k})]
$$

where the sum occurs over all phonon modes, $j$, and wave-vectors $\vec{k}$, and the eigen-vector and frequency of a given mode are denoted by $\vec{e}_{i}(j, \vec{k})$ and $\omega(j, \vec{k})$.

The observed PDOS of sample A at $3.8 \mathrm{~K}$ as measured on FANS is shown in Fig. 3(a). The measurement of the empty can has been subtracted out as background. The phonons have a well-defined cutoff frequency of approximately $41 \mathrm{meV}$. Between 10 and $40 \mathrm{meV}$, six distinct peaks in the density of states are observed at 12, 17.5, 20.5, 24.5, 31.5, and $38 \mathrm{meV}$. Note that the three peaks below $10 \mathrm{meV}$ are not reliable because the scattering at those energies suffers from $\frac{\lambda}{2}$ contamination from the three highest peaks in the PDOS [21].

Since reliable data could not be collected below 10 meV on FANS, an additional measurement of the low-energy phonons was made on DCS with an incident wavelength of 1.8 $\AA$ for Sample A. The background was subtracted out as determined by the intensity at high energy transfers $\left(\mathrm{E}_{i}<\mathrm{E}_{f}\right)$ at $1.5 \mathrm{~K}$, where no inelastic scattering is expected. Fig. 4(a) shows the Q-integrated intensity, $I(\hbar \omega)=\int_{1.5 A^{-1}}^{6.5} I(Q, \hbar \omega) d Q$, at $\mathrm{T}=1.5 \mathrm{~K}, 100 \mathrm{~K}, 200 \mathrm{~K}$, and 300 $\mathrm{K}$. Two peaks are evident at $300 \mathrm{~K}$, one at $8.5 \mathrm{meV}$ and the other at $5.5 \mathrm{meV}$. As the temperature is lowered, the intensity corresponding to both peaks decreases due to a reduced 
thermal population of phonons, and the peak at $5.5 \mathrm{meV}$ becomes too weak to resolve at 1.5 $\mathrm{K}$. The peak at $8.5 \mathrm{meV}$ continually hardens, shifting to $\approx 9.3 \mathrm{meV}$, as the temperature is lowered to $1.5 \mathrm{~K}$. The peaks in the PDOS above $10 \mathrm{meV}$ are difficult to observe using DCS. This is because measured phonon intensity decreases as the inverse of $\hbar \omega$ and the flux of neutrons on DCS is much weaker than that of FANS. Although there is an observed peak at $\hbar \omega=17 \mathrm{meV}$, there is also a known detector spurion for DCS there, so it is much more reliable to trust the data for $\hbar \omega>10 \mathrm{meV}$ on FANS. Fig. 4(b) shows the energy-integrated intensity, $I(Q)=\int_{-11 m e V}^{-3} I(Q, \hbar \omega) d \hbar \omega$, also at T=1.5 K, $100 \mathrm{~K}, 200 \mathrm{~K}$, and $300 \mathrm{~K}$. Fig. 4(b) also shows a scaled down $I(\mathrm{Q}, \hbar \omega=0)$. Since for inelastic scattering from phonons, $I(Q)$ is approximately proportional to $Q^{2} I(Q, \hbar \omega=0)$ [22], this shows that the inelastic scattering over this energy range is indeed from phonons.

The PDOS of sample A was measured below $(3.8 \mathrm{~K})$ and above $(13 \mathrm{~K}) \mathrm{T}_{c}$ on FANS, as shown in Fig. 5. No clear difference in the PDOS could be detected across the superconducting transition given the experimental statistics. A similar lack of change of the PDOS was reported for LaFeOAs [23]. The PDOS of sample B is also shown in Fig. 5 at 3.8 and $100 \mathrm{~K}$. The similarity of the measurements of samples $\mathrm{A}$ and $\mathrm{B}$ at $3.8 \mathrm{~K}$ indicates that the inelastic scattering from Fe-based impurities is minimal since sample A has a higher impurity content than sample B. The comparison between $3.8 \mathrm{~K}$ (orthorhombic) and $100 \mathrm{~K}$ (tetragonal) data shows that the spectral weight of the phonons is relatively unaffected by the structural phase transition.

The bare PDOS according to the calculation of Subedi et al. [5] for stoichiometric FeSe is shown in Fig. 3(b) along with the bare partial PDOS of Fe and Se. The calculation has been convoluted with the instrumental resolution function of FANS with the PG monochromator and the PDOS of Fe and Se have been weighted by $\frac{\sigma_{i}}{m_{i}}$. It should be noted that the bare PDOS and the neutron-weighted PDOS are different in that the neutron-weighted PDOS is weighted by the squared moduli of eigenvectors, which will cause a small difference in the observed peak heights of the two quantities since Fe and Se have difference atomic masses, but the peak positions are expected not to be very sensitive to this difference [22, 24]. According to the calculation, there are four main bands of phonons. The highest energy band is predicted to be a doublet, as we observe, with peaks at $39 \mathrm{meV}$, which is close to the observed value, and $36 \mathrm{meV}$, which is $45 \mathrm{meV}$ higher than the observed value. The second highest band is centered at $28 \mathrm{meV}$, which we also observe at $24.5 \mathrm{meV}$. The third highest 
band is at $18 \mathrm{meV}$; we observe a split band with peaks at 17.5 and $20.5 \mathrm{meV}$ although the calculation shows that there should be a shoulder at the lower energy transfer so the observed splitting is not unreasonable. The same bare PDOS calculation is also shown in Fig. 4(c) for the lower energy phonons. The calculation has been corrected for the Bose factor and the $\frac{1}{\hbar \omega}$ term in the scattering cross section for phonons [22] and also convoluted with the instrument resolution function so that it can be compared more directly with observed spectra. All temperature dependence emanates from the Bose factor (the same bare PDOS is used for all temperatures). The agreement between the observed and predicted peaks is quite good at $1.5 \mathrm{~K}$ where the calculation is most apt. The calculation predicts a peak around $10 \mathrm{meV}$, which is consistent with the experimentwithin $1 \mathrm{meV}$ at $1.5 \mathrm{~K}$. The main difference between the calculation and the measurement is that a peak is observed at $5 \mathrm{meV}$ at higher temperatures, whereas the calculation predicts the peak between 2 and $3 \mathrm{meV}$. Thus all bands of phonons predicted by the calculation are observed in the experiment with only small differences in individual peaks.

The main conclusion of the phonon calculations is that standard electron-phonon coupling cannot account for a $\mathrm{T}_{c}$ of even $1 \mathrm{~K}$. The closeness of the observed and calculated phonon cut-off frequencies along with the observation of all predicted modes with only small shifts in energy support this conclusion. We note that similar electron-phonon calculations and subsequent neutron measurements find the same conclusion for LaFeAsO [23, 24]. On the other hand, PDOS measurements and electron-phonon coupling calculations agree in explaining the superconductivity in other systems such as $\mathrm{MgB}_{2}$ [22, 25].

Finally, we note that recently a magnetic resonance has been observed by inelastic neutron scattering measurements of a powder sample of $\mathrm{Ba}_{0.6} \mathrm{~K}_{0.4} \mathrm{Fe}_{2} \mathrm{As}_{2}$ [26], a result that suggests that spin fluctuations in the superconducting state could be a universal feature of cuprate, heavy fermion, and iron superconductors. If the resonant energy, $\hbar \omega_{r}$, scales as 4.2 times $\mathrm{T}_{c}$ as observed in that $\mathrm{Ba}_{0.6} \mathrm{~K}_{0.4} \mathrm{Fe}_{2} \mathrm{As}_{2}$, then $\hbar \omega_{r}$ should be approximately $2.9 \mathrm{meV}$ for FeSe. We could not observe any magnetic resonance in our experiments on DCS, which is similar to the findings for $\mathrm{LaO}_{0.87} \mathrm{~F}_{0.13} \mathrm{FeAs}$ made on the same instrument [24]. 


\section{CONCLUSION}

In conclusion, we used inelastic neutron scattering techniques as an investigation into the new $\mathrm{FeSe}_{1-x}$ superconductors. We did not observe a significant change in the PDOS across the superconducting transition temperature, nor did we observe a large change across the orthorhombic-tetragonal phase transition. Our results are in general support of the

calculations of Subedi et al. [5], which suggests that their calculation of electron-phonon coupling is reasonable.

\section{ACKNOWLEDGEMENTS}

The authors would like to thank T. J. Udovic, C. M. Brown, P. M. Gehring, V. Kazimirov, M. Kofu and S. Ji for helpful discussions and assistance and are grateful to A. Subedi for providing the PDOS calculations. This work utilized facilities supported in part by the National Science Foundation under Agreement No. DMR-0454672.

[1] F.-C. Hsu, J.-Y. Luo, K.-W. Yeh, T.-K. Chen, T.-W. Huang, P. Wu, Y.-C. Lee, Y.-L. Huang, Y. Chu, D.-C. Yan, et al., Proc. Natl. Acad. Sci. USA 105, 14262 (2008).

[2] K.-W. Yeh, T.-W. Huang, Y.-L. Huang, T.-K. Chen, F.-C. hsu, P. Wu, Y.-C. Lee, Y.-Y. Chu, C.-L. Chen, J.-Y. Luo, et al., Europhys. Lett. 84, 37002 (2008).

[3] M. Fang, H. Pham, B. Qian, T. Liu, E. Vehstedt, Y. Liu, L. Spinu, and Z. Mao, Phys. Rev. B 78, 224503 (2008).

[4] Y. Mizuguchi, F. Tomioka, S. Tsuda, T. Yamaguchi, and Y. Takano, Appl. Phys. Lett. 93, 152505 (2008).

[5] A. Subedi, L. Zhang, D. Singh, and M. Du, Phys. Rev. B 78, 134514 (2008).

[6] K.-W. Lee, V. Pardo, and W. E. Pickett, Phys. Rev. B 78, 174502 (2008).

[7] T. M. McQueen, Q. Huang, V. Ksenofontov, C. Felser, Q. Xu, H. Zandbergen, Y. Hor, J. Allred, A. Williams, D. Qu, et al., Phys. Rev. B 79, 014522 (2009).

[8] H. Kotegawa, S. Masaki, Y. Awai, H. Tou, Y. Mizuguchi, and Y. Takano, J. Phys. Soc. Jpn. 77, 113703 (2008). 
[9] W. Bao, Y. Qiu, Q. Huang, M. A. Green, P. Zajdel, M. R. Firzsimmoons, M. Zhernenkov, M. Fang, B. Qian, E. K. Vehstedt, et al., arXiv:0809.2058 (2008).

[10] R. Paul, R. Lindstrom, and A. Heald, J. Radioanal. Nucl. Chem. 215, 63 (1997).

[11] R. Paul, The Analyst 122, 35(R) (1997).

[12] S. Margadonna, Y. Takabayashi, M. McDonald, K. Kasperkiewicz, Y. Mizuguchi, Y. Takano, A. Fitch, E. Suarde, and K. Prassides, Chem. Commun. p. 5607 (2008).

[13] S. Li, C. de la Cruz, Q. Huang, Y. Chen, J. Lynn, J. Hu, Y.-L. Huang, F.-C. Hsu, K.-W. Yeh, M.-K. Wu, et al., Phys. Rev. B 79, 054503 (2009).

[14] A. C. Larson and R. B. V. Dreele, Los Alamos National Laboratory Report: LAUR 86-748 (2004).

[15] B. H. Toby, J. Appl. Cryst. 34, 210 (2001).

[16] J. Copley, D. Neumann, and W. Kamitakahara, Canadian Journal of Physics 73, 763 (1995).

[17] J. Copley and J. Cook, Chem. Phys. 292, 477 (2003).

[18] http://www.ncnr.nist.gov/dave.

[19] T. Udovic, C. Brown, J. Leao, P. Brand, R. Jiggetts, R. Zeitoun, T. Pierce, I. Peral, J. Copley, Q. Huang, et al., Nucl. Instrum. Methods Phys. Res., Sect. A 588, 406 (2008).

[20] Here $\mathrm{G}(\omega)$ is defined consistent with the generalized density of states (GDOS) in Ref. [22]. Note that the well-known $\frac{Q^{2}}{\omega}$ term in the formula for observed inelastic neutron scattering intensity from phonons is cancelled out by the trajectory of Q- $\omega$ space probed by FANS as discussed in Ref. [16].

[21] The half-lambda contamination is the result of the second order Bragg reflection of incident neutrons through the monochromator that have half the wavelength and thus four times the incident energy as the principally reflected neutrons. This leads to peaks that appear in the measurement at the energy transfer $\hbar \omega$, but actually result from the features that have a real energy transfer of $4 \hbar \omega+3.6 \mathrm{meV}$.

[22] R. Osborn, E. Goremychkin, A. Kolesnikov, and D. Hinks, Phys. Rev. Lett. 87, 017005 (2001).

[23] A. Christianson, M. Lumsden, O. Delaire, M. Stone, D. Abernathy, M. McGuire, A. Sefat, R. Jin, B. Sales, D. Mandrus, et al., Phys. Rev. Lett. 101, 157004 (2008).

[24] Y. Qiu, M. Kofu, W. Bao, S.-H. Lee, Q. Huang, T. Yildirim, J. Copley, J. Lynn, T. Wu, G. Wu, et al., Phys. Rev. B 78, 052508 (2008).

[25] T. Yildirim, O. Gulseren, J. Lynn, C. Brown, T. Udovic, Q. Huang, N. Rogado, K. Regan, 
M. Hayward, J. Slusky, et al., Phys. Rev. Lett. 87, 037001 (2001).

[26] A. Christianson, E. Goremychkin, R. Osborn, S. Rosenkranz, M. D. Lumsden, C. Malliakas, L. S. Todorov, H. Claus, D. Chung, M. G. Kanatzidis, et al., Nature 456, 930 (2008). 
Table I: Rietveld Refinement Results for Sample B as measured on BT-1.

\begin{tabular}{|c|c|c|c|c|c|}
\hline \multicolumn{6}{|c|}{$\begin{array}{l}\mathrm{T}=298 \mathrm{~K}, \mathrm{P} 4 / \mathrm{nmms} \\
\chi^{2}=1.84, \mathrm{R}_{W p}=6.73 \%, \mathrm{R}_{p}=5.37 \% \\
\mathrm{a}=3.7724(1) \AA, \mathrm{c}=5.5217(1) \AA\end{array}$} \\
\hline Atom site & $\mathrm{x}$ & $\mathrm{y}$ & $\mathrm{z}$ & $\mathrm{U}_{I S O}\left(10^{-2} \AA^{2}\right)$ & occ. \\
\hline $2 \mathrm{a}$ & 0 & 0 & 0 & $1.14(5)$ & 1 \\
\hline $2 \mathrm{c}$ & 0 & $\frac{1}{2}$ & $0.2673(2)$ & $1.27(6)$ & $1.0030(5)$ \\
\hline \multicolumn{6}{|c|}{$\begin{array}{l}\mathrm{T}=6 \mathrm{~K}, \text { Cmme } \\
\chi^{2}=3.34, \mathrm{R}_{W p}=8.19 \%, \mathrm{R}_{p}=6.34 \% \\
\mathrm{a}=5.3081(1) \AA, \mathrm{b}=5.3354(1) \AA, \mathrm{c}=5.4879(1) \AA\end{array}$} \\
\hline Atom site & 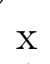 & $\mathrm{y}$ & Z & $\mathrm{U}_{I S O}\left(10^{-2} \AA^{2}\right)$ & occ. \\
\hline $4 \mathrm{a}$ & 4 & 0 & 0 & $0.27(3)$ & 1 \\
\hline $\mathrm{Se}$ & $0^{4}$ & $\frac{1}{4}$ & $0.7352(2)$ & $0.25(6)$ & $0.988(6)$ \\
\hline
\end{tabular}




\section{Figure Captions:}

Fig. 1:

(a) X-ray diffraction patterns of Samples A and B. Reflections are labeled in tetragonal (P4/nmm) notation. Positions of possible impurity peaks (very weak) are denoted with the symbols $\beta$, ${ }^{*}$, and $\nabla$, for $\beta$-FeSe, $\mathrm{Fe}_{3} \mathrm{O}_{4}$, and $\mathrm{Fe}_{3} \mathrm{Si} / \mathrm{Fe}$, respectively. (b) Electrical resistivity of Sample B.

Fig. 2:

Observed $($ red + ) and fitted (solid green) powder neutron diffraction pattern of Sample $\mathrm{B}$ as measured on BT-1 at (a) $\mathrm{T}=298 \mathrm{~K}$ and (b) $\mathrm{T}=4 \mathrm{~K}$. The regions of missing data are masked and correspond to aluminum reflections. The difference curve is shown at the bottom in solid black and the calculated positions of the reflections are shown. The insets show the clear splitting of reflections at $6 \mathrm{~K}$ and indicate the lower symmetry of the low temperature phase. The inclusion of a weak NiAs-phase of FeSe was included to slightly improve the fits.

Fig. 3:

(a) The measured PDOS of Sample A at 3.8 K. Data taken with the PG (002) monochromator is shown in red, whereas data taken with the $\mathrm{Cu}$ (220) monochromator is shown in blue. The empty can background has been subtracted from both measurements. The $\mathrm{Cu}$ data is multiplied by a constant term to put the same data on the scale with the PG data. The reason that there is a larger dip in the $\mathrm{Cu}$ intensity than the PG intensity around $35 \mathrm{meV}$ is that the energy resolution of the $\mathrm{Cu}$ monochromator is superior at that energy transfer to that of the PG monochromator. The three peaks below $10 \mathrm{meV}$ are marked

for their $\frac{\lambda}{2}$ contamination. (b) The partial PDOS of Fe and Se and the total PDOS, as described in the text. The error bars in (a) and later figures represent the $\pm 1 \sigma$ statistical uncertainty.

Fig. 4:

(a) $I(\hbar \omega)$, in (a), and $\mathrm{I}(\mathrm{Q})$, in (b), for Sample A at T=1.5 K, $100 \mathrm{~K}, 200 \mathrm{~K}$, and $300 \mathrm{~K}$. $I(Q, \hbar \omega=0)$, i.e. the diffraction pattern, has been scaled down and is shown at the bottom. 
The particularly strong Bragg reflection at $\mathrm{Q}=3.1 \AA^{-1}$ is marked with an asterisk for its contamination from the $\mathrm{Al}$ sample can and also $\mathrm{Fe} / \mathrm{Fe}_{3} \mathrm{Si}$ impurities. (c) The expected inelastic scattering spectrum based on the PDOS calculation.

Fig. 5:

A comparison of the measured PDOS of Sample A at $3.8 \mathrm{~K}$ and $13 \mathrm{~K}$ and Sample B at 3.8 K and 100 K. Data are intentionally offset for clarity. 

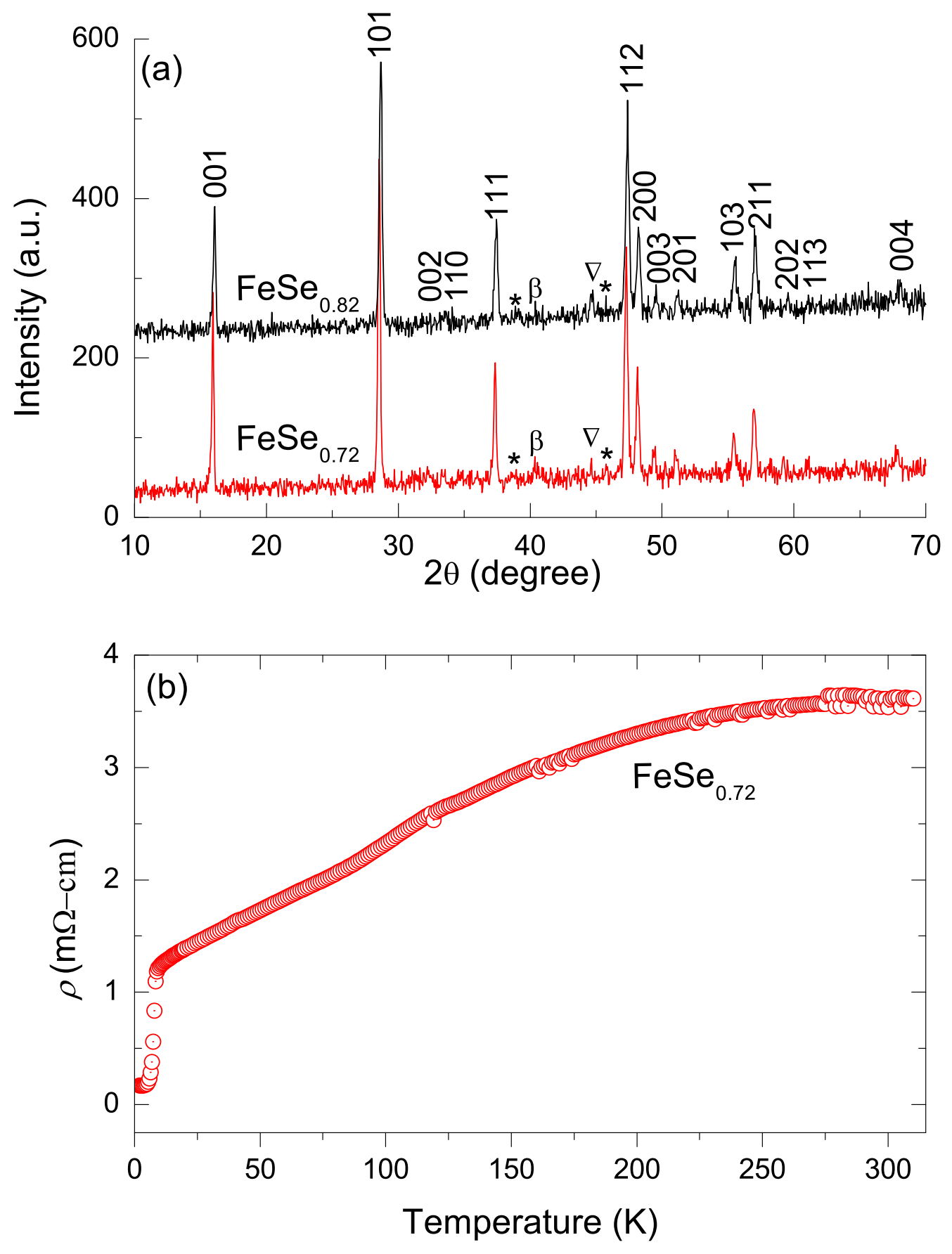

Phelan et al., Fig. 1 

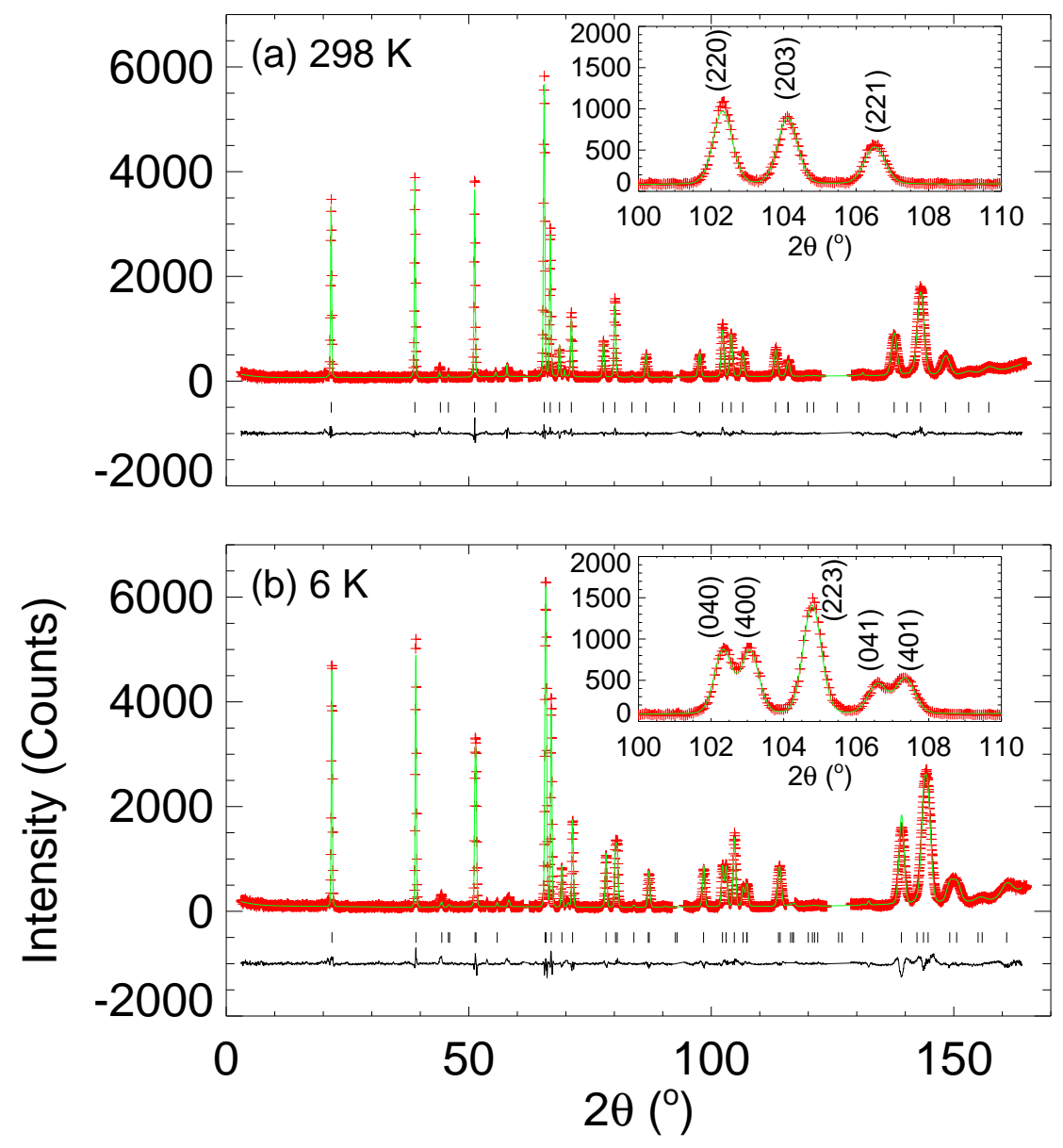

Phelan et al., Fig. 2 

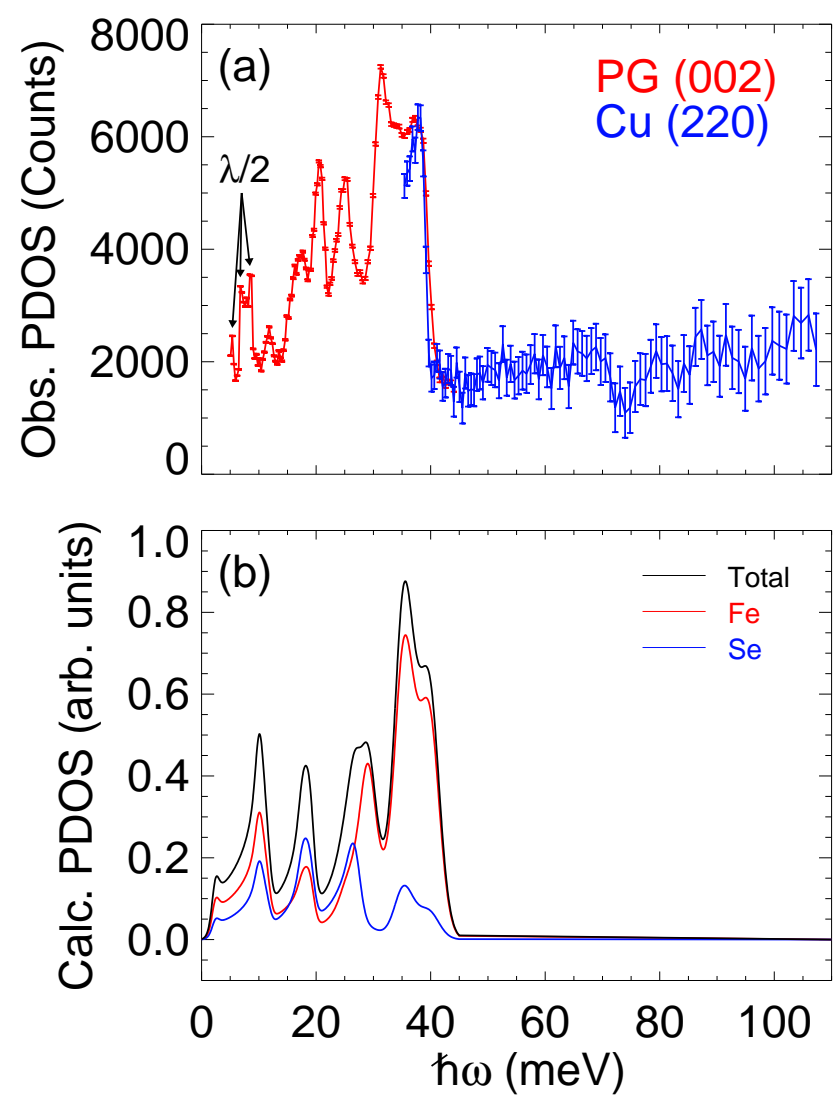

Phelan et al., Fig. 3 

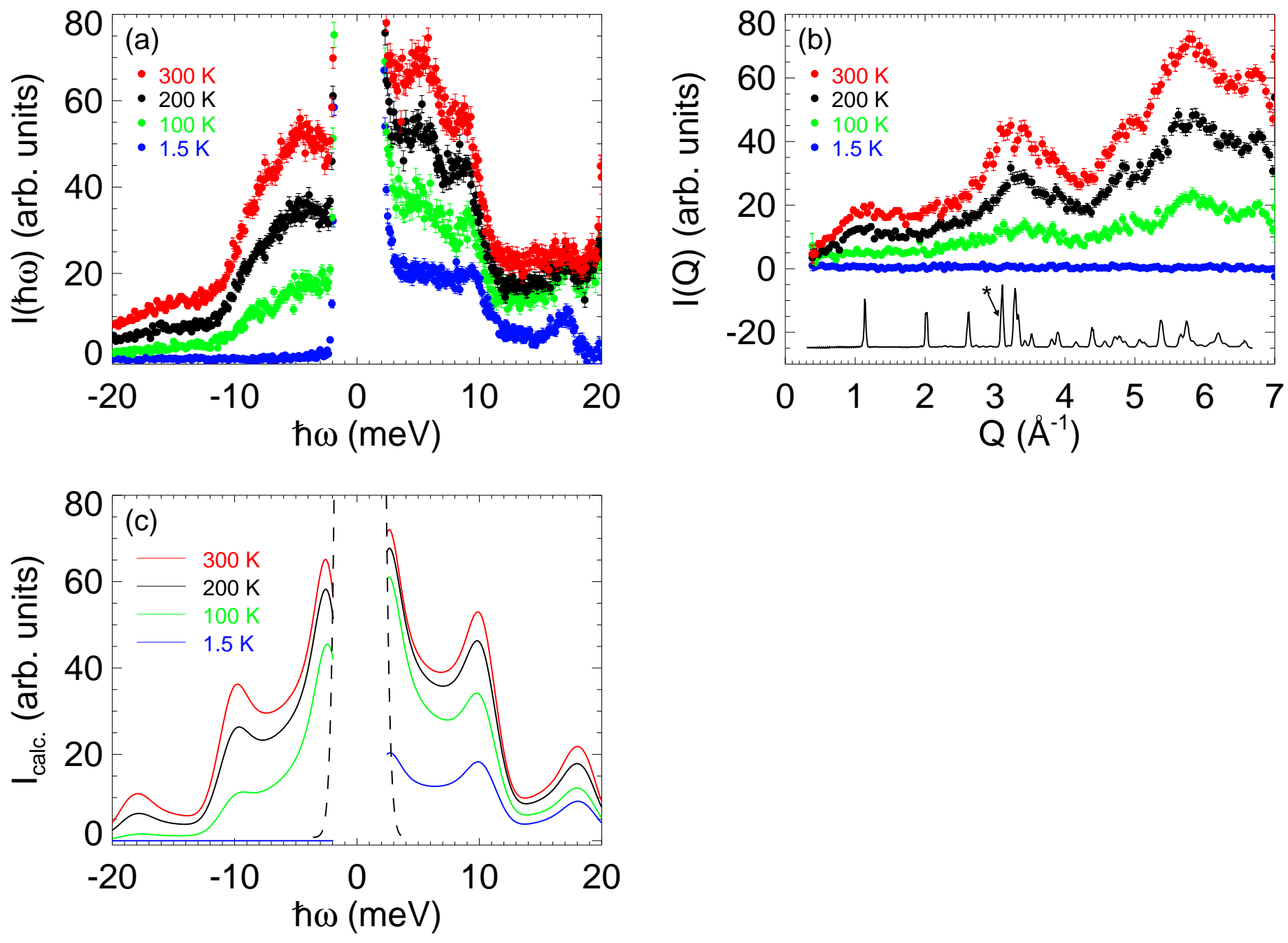

Phelan et al., Fig. 4 


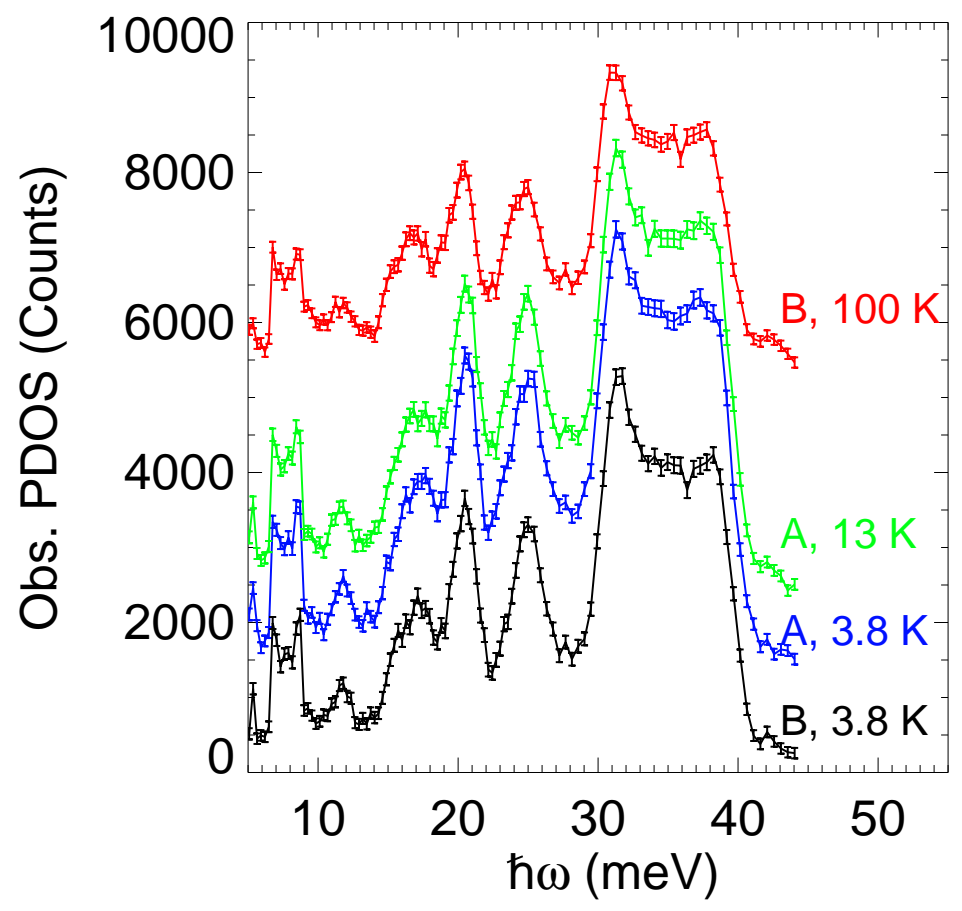

Phelan et al., Fig. 5 